Expert Opinion on Investigational Drugs

EDITORIAL

\title{
The future of obesity: new drugs versus lifestyle interventions
}

\author{
André J Scheen \\ Division of Diabetes, Nutrition \& Metabolic Disorders \\ Unit of Clinical Pharmacology \\ Academic Hospital of Liège \\ University of Liège \\ CHU Sart Tilman (B35) \\ B 4000 Liège \\ Belgium \\ andre.scheen@chu.ulg.ac.be
}

Phone : 32-4-3667238

Fax : 32-4-3667068

\begin{abstract}
Background : Obesity causes serious medical complications and impairs quality of life. However, its management remains challenging.

Objective : To assist health professionals who counsel patients with overweight or obesity by discussing the possible add-on value of new drugs over lifestyle interventions.
\end{abstract}


Methods : Critical analysis of the available evidence of the long-term efficacy of diet and exercise and/or anti-obesity agents such as orlistat, sibutramine and rimonabant.

Results/Conclusion : Lifestyle interventions remain the cornerstone of the treatment of obesity, but adherence is poor and long-term success is modest. Pharmacological agents may be useful adjuncts for improving weight loss and maintenance and health outcomes, and should be continued in good responders. Drug therapy and lifestyle intervention are not opponent strategies, but should probably be combined to tackle obesity.

Key-words : Diet, drug therapy, lifestyle, obesity, weight loss 


\section{Overview}

The term epidemic is commonly used to describe the rapidly increasing rate of obesity worldwide [1]. The challenge is to use our better understanding of the regulation of energy balance to develop strategies to address this obesity epidemic [2]. Obesity arises as a consequence of how the body regulates energy intake, energy expenditure, and energy storage. It is tempting to try to identify one or two environmental factors that are most responsible for weight gain in the population. On the one hand, our current food supply now is one that is high in fat, energy dense, and high in sugar. On the other hand, our physical activity environment has changed largely due to technological advances that make it unnecessary to be physically active in our daily lives. The major challenge becomes to identify the factors in the environment that can be changed to prevent positive energy balance and stop the gradual weight gain of the population (general societal approach) and to treat obesity in those already affected (individual medical strategy). These simple observations support the use of so-called lifestyle interventions for both prevention and treatment of obesity [1-3]. Addressing the obesity epidemic and the individual weight management is going to require closer links between the intake and expenditure side of the energy equation, and the involvement of physical exercise advice is certainly an important consideration besides dietary recommendations [2]. Many overweight and obese individuals have tried to lose weight with lifestyle interventions, and most have been successful to some degree. However, few succeed in long-term maintenance of weight loss.

Obesity has been described as the greatest current threat to human health. Therefore, obesity should no longer be viewed as a cosmetic or body-image issue but rather as a chronic disease [1]. The justification for treating obesity is founded on its enormous cost to health services coupled with evidence of the benefits of weight loss. However, there is less welldocumented evidence for benefits of weight loss than for the association between obesity, especially abdominal obesity, and secondary diseases. Nevertheless, there is strong evidence that weight loss can reduce the risk for type 2 diabetes and cardiovascular risk factors [4]. From a health care perspective, addressing overweight and obesity is an important strategy. The first stage in weight management should be an assessment of overall health and compounding risk factors. The approach to weight management depends on the starting body mass index (BMI), 
the presence of comorbidities and the wishes of the patient. The criteria of the US National Institutes of Health or the European Union for the use of pharmacotherapy include a BMI of at least $27.0 \mathrm{~kg} / \mathrm{m}^{2}$ with a persistent comorbidity or a BMI of at least $30.0 \mathrm{~kg} / \mathrm{m}^{2}$ [5].

Despite the plethora of diet books and heavily promoted magic schemes for effortless and rapid weight loss, the escalating epidemic of obesity shows the failure of these approaches [1]. The guidelines recommend that weight-loss and weight-maintenance therapy should be based on a comprehensive weight-management programme, including diet, physical activity, and behavioural therapy; that medical nutrition therapy for weight loss should last at least 6 months with implementation of a weight-maintenance programme after that time; and that individualized goals of weight-loss therapy should be to reduce body weight at an optimal rate of 0.5 to $1 \mathrm{~kg}$ per week for the first 6 months and to achieve an initial weight-loss goal of up to $10 \%$ from baseline $[3,5]$. Guidelines also suggest the addition of a selected pharmacological agent for appropriate overweight or obese adults, who are not attaining or who are unable to maintain clinically important weight loss with dietary and exercise therapy [5], to assist in reducing obesity-related symptoms and overall cardiometabolic risk, and in overweight or obese patients with type 2 diabetes to improve glycaemic control [6]. Although pharmacological therapy can be offered to obese patients who have failed to achieve or maintain their weight loss goals through diet and exercise alone, there needs to be a doctor-patient discussion of the drug's side effects, the lack of long-term safety data, the temporary nature of the weight loss achieved with medications and the cost of long-term treatment, before initiating drug therapy.

The success of any weight management programme must be evaluated over a relatively long period of time [1]. Weight loss is usually short term and cannot be considered the sole criterion of success. Although diet and lifestyle changes remain the cornerstones of therapy of obesity, weight losses are often small, and long-term success is disappointing [7]. A recent systematic review and meta-analysis of weight-loss clinical trials with a minimum 1-year followup showed that weight-loss interventions utilizing a reduced-energy diet and exercise are associated with moderate weight loss at 6 months [8]. Although there is some regain of weight, weight loss can be maintained. However, regular and prolonged professional contact is most important, and the attrition rate is high. Interventions that include food and meal planning 
strategies resulted in a mean weight loss of approximately 5 to $8.5 \mathrm{~kg}$ (5\% to 9\%) from starting weight during the first 6 months, which stabilized at 4.5 to $7.5 \mathrm{~kg}$ at 12 months in completers. The addition of weight-loss medications somewhat enhances weight-loss maintenance. While the pattern of weight loss and maintenance with the use of weight-management medications is similar to diet plus exercise at 6 months among completers, at 24 months participants maintained a mean weight loss of approximately 2 to $5 \mathrm{~kg}$ ( $2 \%$ to $5 \%)$ above diet and exercise interventions [8]. This additional effect of drug therapy resulted in at least a doubling of the success rate, i.e. the proportion of patients who achieved and maintained a reduction of $5 \%$ or $10 \%$ of initial body weight. Although these results might still appear somewhat disappointing, it is now recognized that such modest weight loss could lead to major improvement in cardiometabolic risk factors and long-term health gains [7]. Because weight regulation is complex, and redundant systems protect against perceived starvation, optimal treatment of obesity will likely require combinations of pharmacological therapies, as in other complex diseases such as diabetes mellitus and arterial hypertension. However, the experience with combined drug therapy is still limited for the obesity management, and such combinations will increase the risk of side-effects, the incidence of drug-drug interactions and the overall treatment cost [9].

The only agents currently accepted for long-term therapy by most regulatory agencies are orlistat, a selective inhibitor of gastrointestinal lipase, sibutramine, a selective inhibitor of serotonin and norepinephrine neuronal reuptake, and recently rimonabant, a selective antagonist of CB1 receptors of the endocannabinoid system [10,11]. Drugs generally induce weight loss slowly and may be more appropriate in weight maintenance. However, none of these anti-obesity drugs is a magic bullet to induce involuntary and substantial weight loss. They are most effective when used as ancillary therapy in a well-organised weight-management programme [3]. An individualised diet with an energy deficit of 500-600 kcal is almost universally used in longerterm trials. Therefore drugs for obesity management should not be opposed to lifestyle intervention but instead the two approaches should be combined to reach the goal [12,13].

Most importantly, some patients fail to respond to the anti-obesity medication, and can usually be detected by failure to lose $2 \mathrm{~kg}$ after 4 weeks or $4-5 \mathrm{~kg}$ after 3 months. If this is the case, the drug should be stopped, because long-term success is unlikely. In contrast, if the drug is 
effective it must be continued. Indeed, withdrawal of an effective anti-obesity drug generally leads to rapid weight regain and worsening of cardiometabolic risk factors. A strange issue surrounding drug treatment of obesity is the perception that because patients regain weight when drugs are stopped, the drugs are ineffective. Quite the contrary is true! [9]. This simply means that obesity is a chronic disease with many causes and whose cure is rare. As for any chronic disease (hypertension, hypercholesterolaemia, diabetes), when the medications are discontinued, the disease is expected to recur. Not surprisingly, the argument that medications only work when they are used also applies for drugs prescribed to treat obesity. They are presently comparatively few drugs available for the treatment of overweight patients, and their effectiveness is limited to palliation rather than cure.

Because all medications inherently have more risks than diet and exercise, deciding to use medications should only be done for people where the benefit justifies the risk [7,9]. As an example, rimonabant has to be prescribed to the right patient, i.e. overweight/obese subjects with cardiometabolic risk factors and with no major depressive illness and/or ongoing antidepressive treatment, in order to both maximise efficacy and minimise safety issues [14-16).

As research progresses, it will not be enough for antiobesity agents to demonstrate bodyfat reduction alone, although recent meta-analyses still tended to restrict their analysis to this limited endpoint [10,11]. Instead, antiobesity agents will be required to demonstrate health benefits beyond weight loss, such as improvement in adipocyte metabolism, and thus improvement in glucose metabolism, dyslipidaemia and blood pressure [7]. To this respect, the demonstration of favourable metabolic changes beyond weight loss with the selective CB1 receptor antagonist rimonabant, resulting at least in part from a significant increase in adiponectin levels, is promising $[15,16]$. The development of any new effective antiobesity agent ideally must not only reduce fat mass (adiposity), but also correct fat dysfunction (“adiposopathy") in order to maximize cardiometabolic health [17].

A crucial missing link regarding antiobesity drugs, compared to lipid-lowering agents or antihypertensive medications for instance, is the lack of large randomised controlled trials demonstrating that their chronic use is able to reduce the incidence of weight-associated complications, especially major cardiovascular events, and prolong life expectancy while 
preserving quality of life. However, this evidence is also missing with lifestyle interventions, essentially because of poor long-term compliance [1]. XENDOS (Xenical in the Prevention of Diabetes in Obese Subjects) was the first study demonstrating that an antiobesity agent like orlistat can reduce the progression to diabetes in comparison with lifestyle changes alone (relative risk reduction of 37\%), especially in obese patients with impaired glucose tolerance [18]. However, such, and even greater, prevention effect of type 2 diabetes was also reported with intensive lifestyle management in the Finnish diabetes prevention study, with an impressive $58 \%$ reduction in the incidence of new diabetes in individuals with impaired glucose tolerance, as compared to those receiving standard management. Most interestingly, such improvement persisted several years after interruption of intensive lifestyle intervention [4], whereas the drug effect usually rapidly vanished after stopping the medication, as already discussed. Orlistat also reduced several cardiovascular risk factors, but the XENDOS study was not designed to assess the effect of the drug on cardiovascular outcomes. A long-term large-scale prospective ongoing trial (Sibutramine Cardiovascular and Diabetes Outcome Study, SCOUT) is currently evaluating the impact of sibutramine on cardiovascular outcomes in obese subjects and the long-term riskbenefit profile of the drug [19]. Finally, the ongoing “CRESCENDO” (Comprehensive Rimonabant Evaluation Study of Cardiovascular ENDpoints and Outcomes) trial will assess whether rimonabant can reduce the risk of major cardiovascular events in 17,000 abdominally obese patients with clustering risk factors followed for 5 years. Obviously, these landmark placebo-controlled trials with hard outcomes will be crucial to support the long-term use of antiobesity medications, in addition to lifestyle interventions, as recently reported for bariatric surgery in the Swedish Obese Subjects study [20].

The complexity of body weight regulation presents a substantial challenge to understanding the etiology of obesity and to developing strategies to treat and/or prevent it [1]. At present, no anti-obesity agent has the capacity to spontaneously change the body weight setpoint without any specific dietary restriction. Therefore, drug therapy and lifestyle intervention should not be opposed but rather combined, and biological and behavioural research is critically important to tackle obesity. We must improve our ability to help people make behaviour changes, which is crucial for lifestyle intervention. In addition, we still have much to learn about 
the biological regulation of energy balance and metabolism in order to use the most effective (and safe) pharmacological approaches.

\section{Expert opinion}

Obesity is a multifactorial disease demanding multidisciplinary management. Furthermore, achieving and maintaining a healthy weight is a life-long process. An energyrestricted diet and regular physical activity are the first-line treatment options for overweight and obese individuals to attain clinically meaningful weight loss, reduce obesity-related symptoms and improve clinical outcomes. The effectiveness of the few drugs available for the long-term treatment of overweight patients is limited to palliation of the chronic disease of obesity. Obviously, drugs are not currently the answer for the majority of obese individuals. None of the available agents has the capacity to spontaneously change the body weight set-point without any specific dietary restriction. However, they could help achieving and maintaining effective weight reduction, although not all individuals are good responders to anti-obesity drug therapy. What is required is a better way of identifying patients who may particularly benefit from a specific pharmacological approach, based on the drug's mechanism of action and on the patient's characteristics. Possibly pharmacogenetics will be helpful, at least in some genetically-prone obese individuals.

Discovery of new pharmacological alternatives, leading to a greater efficacy in the promotion of weight reduction without affecting safety, remains a main objective for the treatment of obesity. Any new antiobesity agent ideally must not only reduce fat mass (adiposity), but also correct fat dysfunction (“adiposopathy”), and consequently abnormal glucose metabolism, dyslipidaemia, hypertension and silent inflammation, in order to maximize cardiometabolic health. Because anti-obesity agents should probably be used lifelong, may lead to some adverse events and are rather expensive, it is crucial that they demonstrate their efficacy and safety in longterm placebo-controlled trials with hard endpoints. Unfortunately, such evidence is massively lacking at present time, although a few large trials are underway. Enhanced efficacy for the next generation of anti-obesity drugs combined with wider use of pharmacotherapy certainly promise to reduce obesity related illness, as it is currently the case for other chronic complex diseases such as diabetes, hypertension or dyslipidaemia. However, the 
challenge might be even greater with obesity management because the major influence toward positive energy balance is coming from to our environment, which promotes energy dense food and low level of physical activity. Therefore, without devoting careful attention toward our environment, our society will not succeed in reversing the obesity epidemic. The response of the medical profession to both the prevention and treatment of obesity is still at an early stage. Most probably, our better scientific understanding of the complexity of energy control and of the recently recognized metabolically active adipose tissue would be helping to validate new pharmacological approaches to tackling the huge problem of obesity and related illnesses in the future.

Declaration of interest : AJ Scheen is a consultant for sanofi-aventis, AstraZeneca, GlaxoSmithKline, and has received lecture fees from sanofi-aventis.

\section{Bibliography}

* 1. Haslam DW, James WPT. Obesity. Lancet 2005; 366: 1197-209.

This paper is a nice overview of the growing burden associated to obesity and of the various approaches to prevent and treat the disease.

2. Hill JO. Understanding and addressing the epidemic of obesity : an energy balance perspective. Endocr Rev 2006; 27: 750-61.

3. Scheen AJ. Integrated approach to treatment and prevention. In : Pharmacotherapy of obesity. Options and alternatives (Eds : Hofbauer K.G., Keller U., Boss O.), CRC Press, Boca Raton, Florida, USA, 2004, 449-63.

4. Lindström J, Ilanne-Parikka P, Peltonen M, et al; Finnish Diabetes Prevention Study Group. Sustained reduction in the incidence of type 2 diabetes by lifestyle intervention: follow-up of the Finnish Diabetes Prevention Study. Lancet 2006; 368: 1673-9.

5. Snow V, Barry P, Fitterman N, Qaseem A, Weiss K, for the Clinical Efficacy Assessment Subcommittee of the American College of Physicians. Pharmacologic and surgical management 
of obesity in primary care: a clinical practice guideline from the American College of Physicians. Ann Intern Med 2005; 142: 525-31.

6. 6. Scheen AJ. Current management strategies for coexisting diabetes mellitus and obesity. Drugs 2003; 63: 1165-84.

7. Scheen AJ, Paquot N. Pharmacological treatment of obesity, food intake, and reversal of metabolic disorders. Curr Nutr Food Sci 2007; 3: 123-33.

* 8. Franz MJ, VanWormer JJ, Crain AL, et al. Weight-loss outcomes: a systematic review and meta-analysis of weight-loss clinical trials with a minimum 1-year follow-up. J Am Diet Assoc 2007; 107: 1755-67.

This paper provides an updated systematic review of eighty randomised clinical trials evaluating long-term interventions involving a reduced-energy diet and/or weight-loss medications

* 9. Bray GA. Medical therapy for obesity - Current status and future hopes. Med Clin N Am 2007; 91: 1225-53.

This paper discusses the efficacy and safety of the few available drugs available for the treatment of obesity and presents various new pharmacological approaches in development. 10. Padwal RS, Majumdar SR. Drug treatments for obesity: orlistat, sibutramine, and rimonabant. Lancet 2007; 369: 71-7.

11. Rucker D, Padwal R, Li SK, Curioni C, Lau DCW. Long term pharmacotherapy for obesity and overweight: updated meta-analysis. BMJ 2007; 335: 1194-9.

12. American Dietetic Association. Position of the American Dietetic Association: integration of medical nutrition therapy and pharmacotherapy. J Am Diet Assoc 2003; 103: 1363-70.

13. Wadden TA, Berkovitz RI, Womble LG, et al. Randomized trial of lifestyle modification and pharmacotherapy for obesity. N Engl J Med 2005; 353: 2111-20.

14. Christensen R, Kristensen PK, Bartels EM, Bliddal H, Astrup A. Efficacy and safety of the weight-loss drug rimonabant: a meta-analysis of randomised trials. Lancet 2007; 370: 1706-13. 15. Scheen AJ. Cannabinoid-1 receptor antagonists in type-2 diabetes. Best Pract Res Clin Endocrinol Metab 2007; 21: 535-53. 
16. Van Gaal LF, Pi-Sunyer X, Després JP, Mc Carthy C, Scheen AJ. Efficacy and safety of rimonabant for improvement of multiple cardiometabolic risk factors in overweight/obese patients: pooled 1-year data from the RIO program. Diabetes Care 2008; in press.

17. Bays, H, Blonde L, Rosenson R. Adiposopathy: how do diet, exercise and weight loss drug therapies improve metabolic disease in overweight patients? Expert Rev Cardiovasc Ther 2006; 4: 871-95.

18. Torgerson JS, Hauptman J, Boldrin MN, Sjöström L. Xenical in the Prevention of Diabetes in Obese Subjects (XENDOS) study. A randomised study of orlistat as an adjunct to lifestyle for the prevention of type 2 diabetes in obese patients. Diabetes Care 2004; 27: 155-61.

19. James WPT. The SCOUT study: risk-benefit profile of sibutramine in overweight high-risk cardiovascular patients. Eur Heart J 2005; 7 (suppl L): L44-8.

20. Sjöström L, Narbro K, Sjöström CD, et al; Swedish Obese Subjects Study. Effects of bariatric surgery on mortality in Swedish obese subjects. New Engl J Med 2007; 357: 741-52. 